\title{
Antidepressant activity of ethanolic extract of oleo gum resins of Ferula asafoetida Linn
}

\author{
T Bharath Kumar', Dr. V Jayasankar Reddy², R Rushendran'1, T Mamatha', J Roja', T Roopavani \\ ${ }^{1}$ Department of Pharmacology, Krishna teja pharmacy college, Tirupati, Andhra Pradesh, India \\ 2 Professor, HOD, Department of Pharmacology, Krishna teja pharmacy college, Tirupati, Andhra Pradesh, India \\ ${ }^{3}$ Department of Pharmacognosy, Sree Vidyanikethan Pharmacy College, Tirupati, Andhra Pradesh, India
}

Bharath Kumar T, Jayasankar Reddy V, Rushendran R, Mamatha T, Roja J, Roopavani T. Antidepressant activity of ethanolic extract of oleo gum resins of Ferula asafoetida Linn. J Pre-Clin Clin Res. 2017; 11(1): 50-60. doi: 10.26444/jpccr/75721

\begin{abstract}
Objective. The study was undertaken to evaluate the antidepressant activity of ethanolic extract of Ferula asafoetida oleo gum resins.

Materials and Methods. Five groups of rats (180-200g) and mice (20-30g) of both genders, each group comprising six animals, were used (i.e., normal, positive control, standard, FAEE $200 \mathrm{mg} / \mathrm{kg}$, and FAEE $400 \mathrm{mg} / \mathrm{kg}$ treated groups). Forced swimming test (FST), Tail suspension test (TST), Potentiation of Norepinephrine-Induced Toxicity (PNEIT), Haloperidol- Induced Catalepsy (HIC), and Reserpine-Induced Hypothermia (RIH), were used as the validate models of depression in rodents. The study was confirmed by brain monoamines estimation (i.e. Dopamine, Norepinephrine and 5-HT), MAO levels and invivo antioxidant studies (CAT and SOD).

Results. FAEE treated animals showed a significant and dose dependent effect on a decrease in immobility time in FST, TST, and decrease in catalepsy time in HIC. FAEE and imipramine $(15 \mathrm{mg} / \mathrm{kg})$ showed a significant increase in body temperature in RIH, and also showed a potent lethality in PNEIT. FAEE treated animals showed a significant increase in the levels of brain monoamines, in vivo antioxidants, and a significant decrease in MAO levels.

Conclusion. Results of present study indicate that FAEE has potent antidepressant-like activity, and this effect may be due to the anti-oxidant property of Ferulic acid and umbelliferone, or may be due to neuroprotective activity of other major phytoconstituents, e.g. flavonoids, phenolic acids and polysulfide compounds. To identify the particular compound responsible for the antidepressant-like activity required further molecular level studies.
\end{abstract}

\section{Key words}

antidepressant activity, Ferula asafoetida, models of depression, brain monoamines, antioxidant activity.

\section{INTRODUCTION}

Depression is a common psychiatric disorder that affects a person's mood, physical health and behavior, and is also often associated with suicidal thoughts; there are between 10-20 million suicide attempts every year. It is estimated that about $5.8 \%$ of men and $9.5 \%$ of women experience a depressive episode in their lifetime. According to a World Health Report, about 450 million people suffer from a mental or behavioural disorder. This amounts $12.3 \%$ of the global burden of disease at the present time, which will rise to $15 \%$ by 2020 . According to the Indian survey, about $7 \%$ of the Indian population suffers from mood and anxiety disorders $[1,2]$.

A constant state of depression occurs due to continuous stress or central nervous system (CNS) neurochemical imbalance. Oxidative stress (OS) is considered to be a major factor in the causation of anxiety and depression, and these CNS disorders appear due to imbalance in oxidation-reduction reactions. It is characterized by the reduced ability of the antioxidant defense mechanism of the CNS to efficiently remove the excess of oxygen-derived free radicals, which are known to produce detrimental effects in the CNS. A growing body of evidence suggests that OS causes imbalance between the production of

Address for correspondence: T Bharath Kumar, Department of Pharmacology, Krishna teja pharmacy college

E-mail: bharathdharu@gmail.com

Received: 9 January 2017; accepted: 15 May 2017 oxygen-derived free radicals and the antioxidant ability of neuronal cells and tissues, consequently contributing to the neuropathology and psychiatric diseases, including mild and major depression [3].

Today, a number of synthetic antidepressant drugs are available for treatment of depression; however, their effectiveness was undisputed with the entire range of population suffering from this depressive disorder. Although most of these medications are safe and efficacious, yet in some patients prolonged usage may cause a variety of minor side-effects, such as dry mouth, mydriasis, constipation, sleepiness, fatigue, restlessness and headaches. In some patients, prolonged therapy with fluoxetine may induce serious side-effects, such as suicidal tendencies. Thus, alternative therapies are needed which could be efficacious but produce less side-effects. On the other hand, herbal medicines are widely used across the world due to their wide applicability and therapeutic efficacy, along with the least side-effects and lower price which, in turn, has increased scientific research regarding antidepressant activity [4].

Over the past few decades, the affinity towards herbal drugs has increased the by utilization of traditional medicinal plant to heal some critical diseases. It is proving to be better medicine with respect to synthetic drugs that ensure numerous side-effects in prolonged treatment. In recent years, the focus on plants research has increased worldwide. A large body of evidence has been collected that shows the immense potential of medicinal plants used in various traditional systems [5]. 
A number of scientific investigations have highlighted the importance and the contribution of many plant families used as medicinal plants and they have played a vital role in the development of new drugs. Plant-derived drugs are used to cure mental illness, skin diseases, tuberculosis, diabetes, jaundice, hypertension, cancer, etc. Medicinal plants play an important role in the development of potent therapeutic agents. Plant-derived drugs came into use in modern medicine through the utilization of plant material as indigenous cures in folklore or traditional systems of medicine.

Determining the biological properties of plants used in traditional medicine is helpful to rural communities and informal settlements. Several attempts are currently being undertaken to isolate the active compounds by bioassayguided fractionation from the species that showed high biological activity during screening. Therefore, these scientific investigations may be utilized to develop drugs for these diseases. Further research is needed to isolate the compounds responsible for the observed biological activity.

Therefore, this present study also aims to determine the biological activity of certain plant, i.e., the 'antidepressant activity' of oleo gum resins of 'Ferula asafoetida' by using experimental animal models of depression, i.e., physical inducing (FST and TST) and drug inducing (HIC, RIH and PNIT).

\section{MATERIAL AND METHODS}

Plant material and preparation of extract. The pure form of oleo gum resins of Ferula asafoetida was collected from the local ayurvedic store in January 2016. The plant material was identified and authenticated by Dr. K. Madhava Chetty, Assistant Professor in the Department of Botany at Sri Venkateswara University, Tirupati, Chittoor District, India. The voucher specimen is available in the herbarium file of our department. The dried plant product was coarsely powdered and placed in a soxhlet apparatus, subjected to continuous hot percolation at the temperature $50^{\circ} \mathrm{C}$, using ethanol as the solvent until a clear solvent was observed in the siphon tube. The extract was concentrated, dried and placed into an air tight container.

Preliminary phytochemical estimation. Preliminary phytochemical estimation by using standard procedures was carried out on the Ethanolic extract of Ferula asafoetida for qualitative identification of the phytochemical constituents, e.g., alkaloids, carbohydrates, gums, amino acids, glycosides, steroids, flavanoids and triterpinoids.

Animals. Healthy adult albino mice $(20-30 \mathrm{~g})$ and Wistar albino rats $(180-200 \mathrm{~g})$ were used in the study. Animals were procured from the animal house of our institute and acclimatized to laboratory conditions, i.e. housed in polypropylene cages, $12: 12 \mathrm{hrs}$ light/dark cycle, $24 \pm 2^{\circ} \mathrm{C}$, relative humidity $50 \pm 5 \%$. They were provided with standard feed pellet (Hindustan Lever Ltd., Bangalore, India) and drinking water ad libitum throughout the experimentation period. The animals were fasted $12 \mathrm{hrs}$ prior the experimentation, but allowed free access to water (OECD guidelines 2001). All the animal studies were performed in accordance with the rules, regulations and guidelines of the CPCSEA (Registered No. IAEC/1521/PO/Re/S/2011/CPCSEA).
Acute toxicity studies. Acute toxicity studies of FAEE were carried out in Swiss albino mice weighing 20-30g. The study was performed according to the OECD Guideline No. 423. Five groups of mice with three animals in each group were used. Group I, the negative control, was administered normal saline, and Group II, Vehicle treated, FAEE was administered at doses of 5,50,300, and 2,000 mg/kg b.w p.o., respectively. Signs or symptoms of acute toxicity and mortality rate were observed continuously for the first $4 \mathrm{~h}$, followed by the $24 \mathrm{~h}$; further observation continued up to 14 days after administration.

Experimental protocol. Animals were randomly divided into five groups of six animals each. Group I - Negative Control (Vehicle treated group, p.o.), Group II - Positive control (Disease- induced animals), Group III - Standard (Imipramine $15 \mathrm{mg} / \mathrm{kg}$, p.o.) in FST,TST, RIH, PNEIT models and Fluoxitine $5 \mathrm{mg} / \mathrm{kg}$, p.o in HIC models), Group IV - Low dose of FAEE (200 mg/kg, p.o.). and Group V - High dose of FAEE (400 mg/kg, p.o).

\section{EVALUATION OF ANTIDEPRESSANT ACTIVITY}

Forced Swimming Test (FST). Before performing this test, animals underwent certain training, i.e., mice were forced to swim for $15 \mathrm{mins}$ individually in a vertical Plexiglass cylinder (height: $40 \mathrm{~cm}$; diameter: $18 \mathrm{~cm}$ ) containing fresh water made up to $15 \mathrm{~cm}$, maintained at $25^{\circ} \mathrm{C}$, carried for seven days. After completion of training, the treatment was started.

In the forced swimming test, after a brief spell of vigorous activity, the animals showed a posture of immobility, characterized by floating motionless in the water making only movements sufficient to keep the head above water (Fig. 1). This immobility reflected the state of depression. Each mouse was subjected to this activity $1 \mathrm{hr}$ after administration of the drug. The duration of immobility time was observed for $6 \mathrm{~min}$, and the actual test recording was performed on the 1st, 7 th and 14th day of treatment. After recording immobility time, the mice were removed, wiped with a dry cloth, and allowed to dry before returning to their home cages $[6,7,8]$.

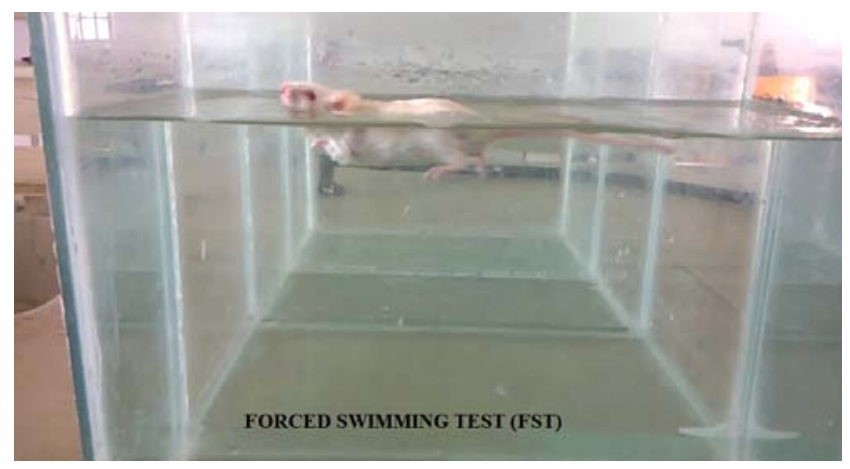

Figure 1. Forced Swimming Test

Tail Suspension Test (TST). Pretraining was given before this test, i.e., mice were suspended on a string held by a metal stand, by an adhesive tape placed $1 \mathrm{~cm}$ from the tip of the tail (Fig. 2). The string was $58 \mathrm{~cm}$ above the table top and the mice suspended for $15 \mathrm{mins}$ for up to 7 days. After completing the training, treatment has been started. In this test, the mice were considered immobile when they hung passively 


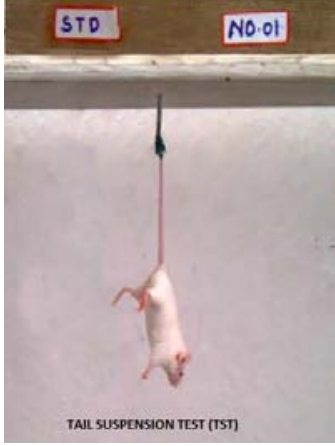

Figure 2. Tail suspension Test

and completely motionless. During the experiment, each animal under test was both acoustically and visually isolated from other animals. This immobility reflected the state of depression. Each mouse was subjected to this activity $1 \mathrm{hr}$ after administration of the drug. The duration of immobility was observed for 6 minutes and recorded on video tapes. Actual test recording was carried out on the $1^{\text {st }}, 7^{\text {th }}$ and $14^{\text {th }}$ day of treatment. On the $14^{\text {th }}$ day after completing the FST, followed by TST, animals were immediately sacrificed, their brains isolated, and subjected to biochemical estimations and histopathological studies $[9,10]$.

Haloperidol-Induced Catalepsy (HIC). Haloperidol ( $1 \mathrm{mg} / \mathrm{kg}$ b.w i.p) was administered to the male wistar albino rats weighing between 180-200g which were arranged in grouped. With the exception of the first group, all groups were treated with haloperidol ( $1 \mathrm{mg} / \mathrm{kg}$ b.w i.p) for 7 days. After the induction of haloperidol, the animals were treated with a standard drug (fluoxitine $5 \mathrm{mg} / \mathrm{kg}$ ) and the test drug (FAEE 200 and $400 \mathrm{mg} / \mathrm{kg}$ ), and catalepsy time was calculated for all groups. The duration of catalepsy was measured after administration of a drug at the intervals of 30,60, 90, 120, 150 , and $180 \mathrm{~min}$. Catalepsy was assessed by means of the standard bar test on every $3^{\text {rd }}, 5^{\text {th }}$ and $7^{\text {th }}$ day of drug treatment. The catalepsy was measured as the time during which the rat maintained an imposed posture, with both front limbs extended and resting on a $4 \mathrm{~cm}$ high wooden bar $(1.0 \mathrm{~cm}$ diameter). The end point of the catalepsy was considered to occur when both the front paws were removed from the bar, or if the animal moved its head in an exploratory manner. A cut-off time of $200 \mathrm{~s}$ was applied $[11,12]$.

Reserpine-Induced Hypothermia (RIH). Reserpine ( $2 \mathrm{mg} / \mathrm{kg}$ b.w i.p) was administered to the Wistar albino rats weighing between 180-200g which were arranged in grouped. With the exception of the first group, all groups were treated with reserpine $\left(2 \mathrm{mg} / \mathrm{kg}\right.$ b.w i.p) on the $6^{\text {th }}$ day after treatment with standard drug (Imipramine $15 \mathrm{mg} / \mathrm{kg}$ b.w p.o) and the test drug (FAEE 200 and $400 \mathrm{mg} / \mathrm{kg}$ b.w p.o). Decrease in temperature of the rat indicated a depression condition which was measured by using rectal temperature at $2 \mathrm{hr}$ intervals for up to $6 \mathrm{hrs}$. The rectal temperature was determined by insertion of a rectal thermometer to a constant depth of $2 \mathrm{~cm}$ before and after administration of reserpine [13].

Potentiation of norepinephrine-induced toxicity. Norepinephrine ( $4 \mathrm{mg} / \mathrm{kg}$ b.w p.o) was administered to the albino mice weighing between 20-25g which were arranged in groups. With the exception of the first group, all the groups were treated with norepinephrine twice a day, and observe for $48 \mathrm{hrs}$. The percentage of mortality rate was observed after treating animals with a standard drug (Imipramine $15 \mathrm{mg} / \mathrm{kg}$ b.w p.o) and extract (FAEE 200 and $400 \mathrm{mg} / \mathrm{kg}$ b.w p.o) to the norepinephrine-treated animals $[14,15]$.

\section{BIOCHEMICAL PARAMETERS OF BRAIN}

Brain monoamine levels estimation. On the last day of the experiment, the mice were sacrificed, whole brain was dissected out and the sub-cortical region (including the striatum) separated. Weigh the tissue and take the $50-75 \mathrm{mg}$ of tissue for homogenate with $5 \mathrm{ml} \mathrm{HCl}$-butanol for about $1 \mathrm{~min}$. The sample was then centrifuged for $10 \mathrm{mins}$ at $2,000 \mathrm{rpm}$. An aliquot supernatant phase $(1 \mathrm{ml})$ was removed and added to the centrifuge tube containing $2.5 \mathrm{ml}$ of heptane and $0.31 \mathrm{ml} \mathrm{HCl}$ of $0.1 \mathrm{M}$. After $10 \mathrm{mins}$ of vigorous shaking, the tube was centrifuged under the same conditions as above in order to separate the 2 phases, and the overlaying organic phase was discarded. The aqueous phase $(0.2 \mathrm{ml})$ was then taken either for 5-HT, NA or DA assay. All steps were carried out at $0^{\circ} \mathrm{C}$. 5 -HT, NA and DA assay was performed using UV/visible spectrophotometer [16, 17].

Estimation of brain MAO levels, Brain mitochondrial fraction was prepared for estimating monoamine oxidase (MAO) activity by using UV/visible spectrophotometer $[18,19]$.

In vivo antioxidant studies - Catalase (CAT). Catalase activity was measured by the method of Aebi (1974). Activity of catalase was expressed as $\mu$ moles of $\mathrm{H}_{2} \mathrm{O}_{2}$ metabolized/mg protein/min [20].

where:

$$
\text { Catalase }=\log \left(\frac{A}{B}\right) \times 2297.3
$$

A: Initial absorbance

B: Final absorbance (after 30 seconds)

Units $=\mu$ moles of $\mathrm{H}_{2} \mathrm{O}_{2}$ consumed $/ \mathrm{min} / \mathrm{mg}$

Superoxide dismutase (SOD). Superoxide dismutase (SOD) activity was determined by the method developed by Kakkar et al. (1984). The SOD level was expressed as units per mg protein [21].

where:

$$
\text { SOD }=\frac{0.025-Y}{Y X 50} \times 100
$$

$\mathrm{Y}=$ Final reading - initial reading

Histopathological studies. The brain tissues were washed immediately with saline and fixed in a $10 \%$ formalin solution. After fixation, the brain tissues were processed in alcoholxylene series and then embedded in paraffin wax. The serial sections were cut at 5-6 $\mu \mathrm{m}$ thickness, and each section was stained with haematoxylin and eosin. The slides were examined under a microscope and photographed [22].

Statistical analysis. Results were presented as mean \pm SEM $(n=6)$. The data was subjected to statistical analysis by Oneway analysis of variance (ANOVA), followed by Dunnett's multiple comparison tests (t-test); ${ }^{a} \mathrm{p}<0.05,{ }^{\mathrm{b}} \mathrm{p}<0.01,{ }^{\mathrm{c}} \mathrm{p}<$ 0.001 were considered as significance, $\mathrm{p}>0.05$ was considered 
as non-significance (ns) which was compared with positive control. All statistical analysis was performed by using Graph pad Prism software (7.01version).

\section{RESULTS}

Preliminary phytochemical estimation. Preliminary phytochemical estimation of FAEE revealed that the following phytochemical constituents were present: carbohydrates, amino acids, alkaloids, phenols, flavonoids, terpenoids and tannins.

Acute toxicity studies. The animals were observed individually 2 hrs after administration of FAEE, periodically during the first $24 \mathrm{hrs}$ and daily thereafter for a total 14 days. There was no change in skin, fur, eyes, mucous membrane, respiratory, circulatory, nervous systems, behaviour pattern and body weights of the rats during the 14 days.

\section{HISTOPATHOLOGICAL STUDIES}

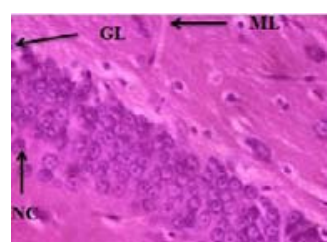

Group I (Normal)

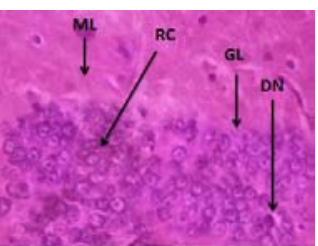

Group III (Standard)

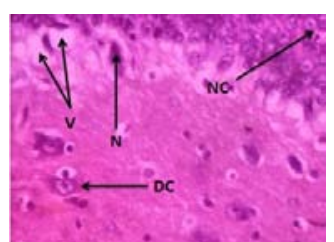

Group II (Positive control)

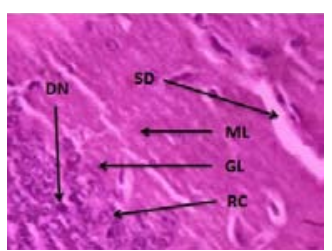

Group IV (Test-1)

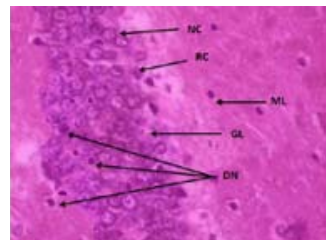

Group V (Test-2)

Figure 3. Effect of FAEE on Hippocampus region of brain in FST and TST performed animals.

Fig 3 (Normal) Transverse section of hippocampus showing normal histoarchitecture (H\&E 40x). Fig 3 (positive control) Transverse section of hippocampus showing the neuronal necrosis, mild degenerative neurons and vacuolization (H\&E 40x). Fig 3 (standard) Transverse section of hippocampus showing almost normal histo-architecture, slight regenerative changes and degenerative neurons (H\&E 40x). Fig 3 (Test-1) Transverse section of hippocampus showing the regenerative changes, some degenerated neurons and slight structural changes (H\&E 40x). Fig 3 (Test-2) Transverse section of hippocampus showing almost normal histoarchitecture but rare degenerative neurons and regenerative changes (H\&E 40x). (GL granular layer, NC Normal cell, DC degenerative changes, $V$ vacuolization, DN degenerated neuron $\mathrm{ML}$ molecular layer, $\mathrm{N}$ necrosis $\mathrm{RC}$ regenerative changes SD structural damage).
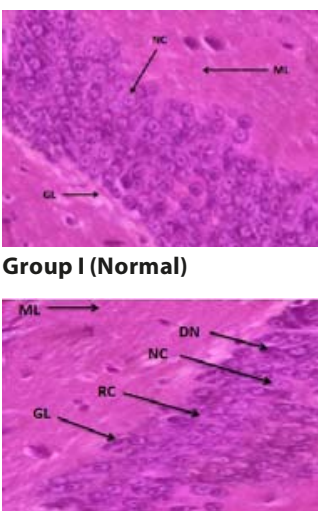

Group III (Standard)
Group I (Normal)

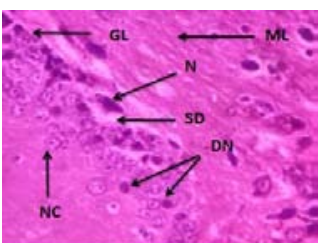

Group II (Positive control)

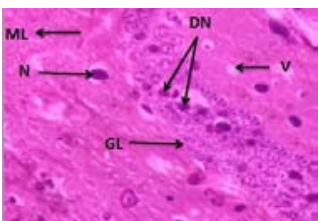

Group IV (Test-1)

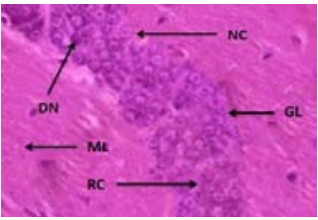

Group V (Test-2)

Figure 4. Effect of FAEE on Hippocampus region of brain in HIC performed animals. Fig 4 (Normal) Transverse section of hippocampus showing normal histo-architecture (H\&E 40x). Fig 4 (Positive control) Transverse section of hippocampus showing the neuronal necrosis, mild degenerative neurons, vacuolization and decreased in cell density H\&E 40x). Fig 4 (Standard) Transverse section of hippocampus showing almost normal histo-architecture, slight degenerated neurons and regenerative changes (H\&E 40x). Fig 4 (Test-1) Transverse section of hippocampus showing degenerated neurons, vacuolization and neuronal necrosis(H\&E 40x). Fig 4 (Test2) Transverse section of hippocampus showing almost normal neuronal cytoarchitecture, but rare regenerative changes and degenerative neurons (H\&E 40x). (GL granular layer, NC Normal cell, DC degenerative changes, V vacuolization, DN degenerated neuron ML molecular layer, $\mathrm{N}$ necrosis $\mathrm{RC}$ regenerative changes SD structural damage).

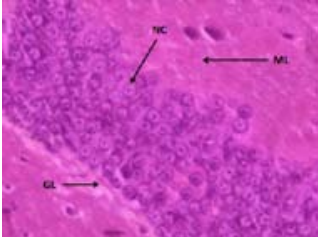

Group I (Normal)

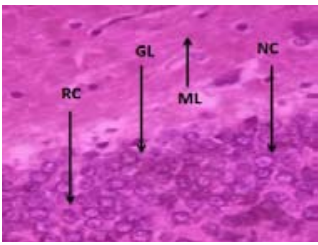

Group III (Standard)

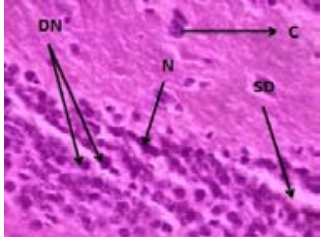

Group II (Positive control)

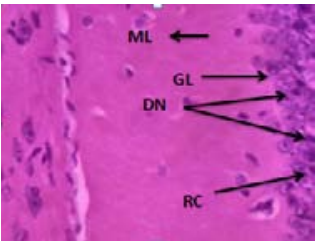

Group IV (Test-1)

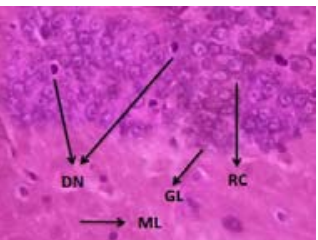

Group V (Test-2)

Figure 5. Effect of FAEE on Hippocampus region of brain in RIH performed animals. Figure 5 (Normal) Transverse section of hippocampus showing normal histoarchitecture (H\&E 40x). Figure 5 (positive control) Transverse section of hippocampus showing congestion, neuronal necrosis, degenerative neurons and structural damages (H\&E 40x). Figure 5 (standard) Transverse section of hippocampus showing almost normal histo-architecture (H\&E 40x). Figure 5 (Test-1) Transverse section of hippocampus showing slight regenerative changes and some degenerated neurons (H\&E 40x). Figure 5 (Test-2) Transverse section of hippocampus showing almost normal neuronal cyto-architecture rare degenerative neuron and regerative changes (H\&E 40x). (GL granular layer, NC Normal cell, DC degenerative changes, $\mathrm{V}$ vacuolization, DN degenerated neuron $\mathrm{ML}$ molecular layer, $\mathrm{N}$ necrosis RC regenerative changes SD structural damage). 


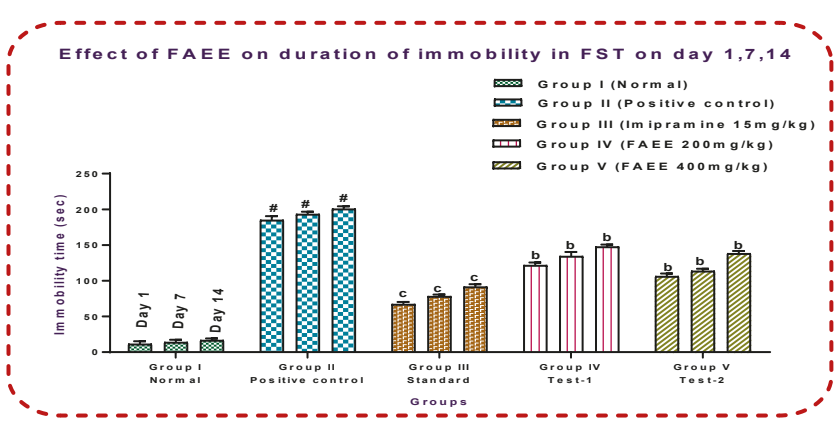

Graph 1. Effect of FAEE on duration of immobility in FST on day $1,7,14$.

All values were expressed as mean \pm SEM, $(n=6)$. Statistical significance: $\mathrm{aP}<0.05$ $\mathrm{bP}<0.01, \mathrm{CP}<0.001$ was compared with positive control (One way ANOVA followed by Dunnett's multiple comparisons test)

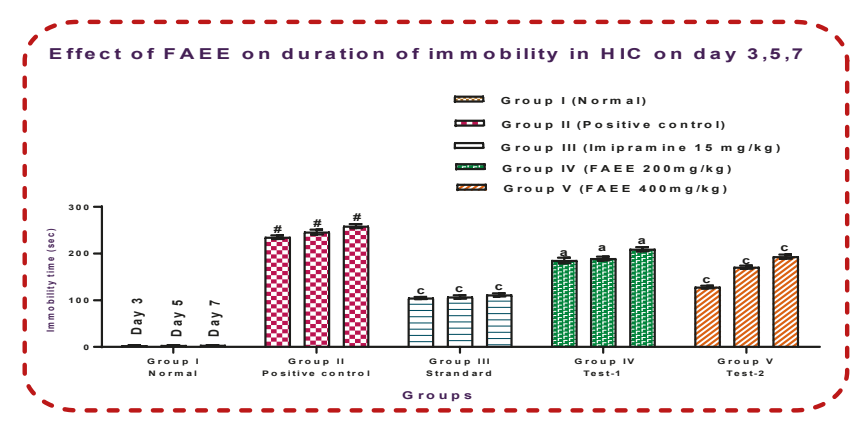

Graph 3. Effect of FAEE on duration of catalepsy in HIC on day 3,5,7.

All values were expressed as mean \pm SEM, $(n=6)$. Statistical significance: $a P<0.05$, $\mathrm{bP}<0.01, \mathrm{cP}<0.001$ was compared with positive control (One way ANOVA followed by Dunnett's multiple comparisons test)

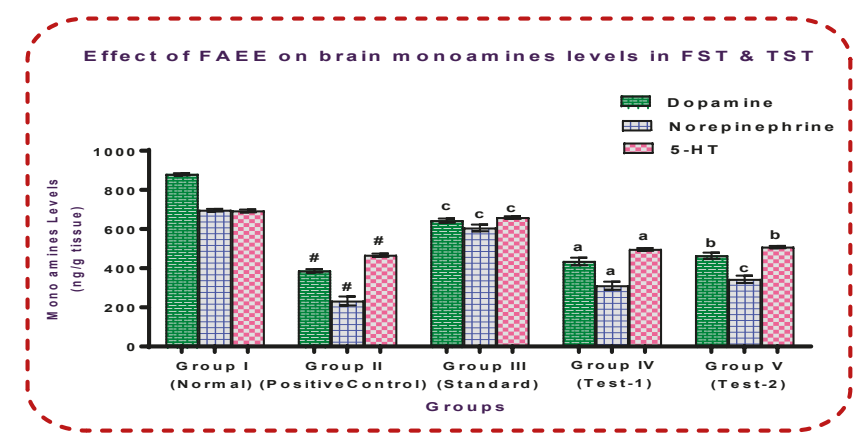

Graph 5. Effect of FAEE on brain monoamines levels in FST and TST

All values were expressed as Mean $\pm \operatorname{SEM}(n=6)$. The data was analysised by One way ANOVA using Dunnett's multiple comparison tests; Significance at aP $<0.05$, $b P<0.01, c P<0.001$ Non Significance (ns) at $P>0.05$ Vs positive control.

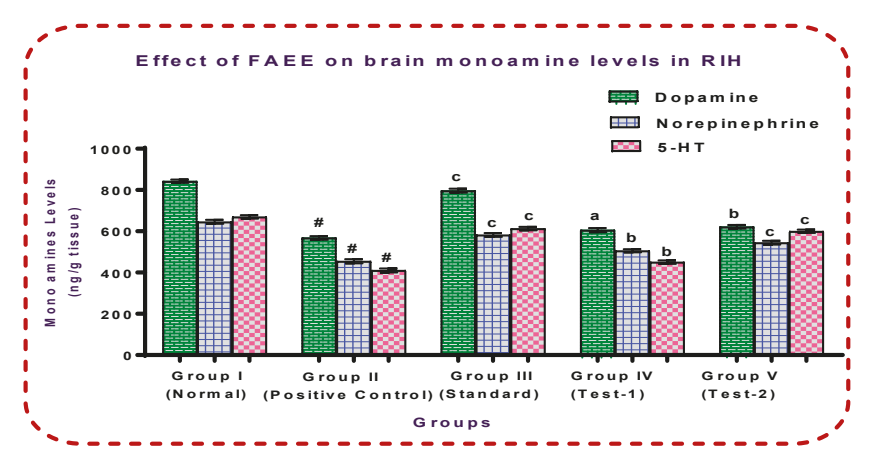

Graph 7. Effect of FAEE on brain monoamines levels in RIH.

All values were expressed as Mean $\pm \operatorname{SEM}(n=6)$. The data was analysised by One way ANOVA using Dunnett's multiple comparison tests; Significance at $\mathrm{aP}<0.05, \mathrm{bP}<0.01$, $c P<0.001$ Non Significance (ns) at $\mathrm{P}>0.05$ Vs positive control.

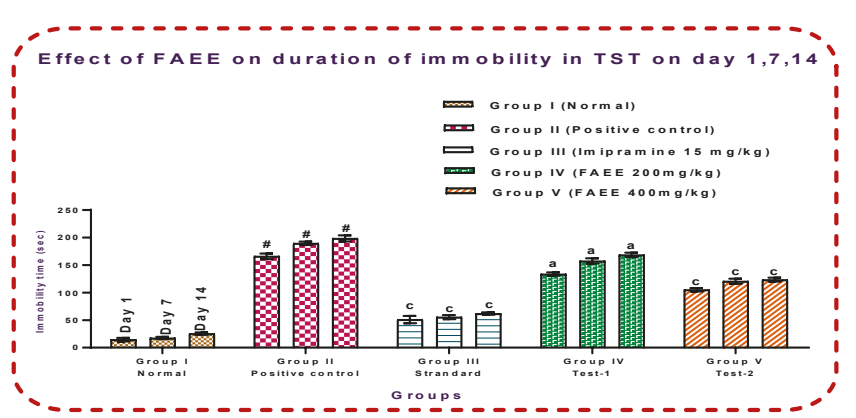

Graph 2. Effect of FAEE on duration of immobility in TST on day $1,7,14$.

All values were expressed as mean \pm SEM, $(n=6)$. Statistical significance: $\mathrm{PP}<0.05$ $\mathrm{bP}<0.01, \mathrm{cP}<0.001$ was compared with positive control (One way ANOVA followed by Dunnett's multiple comparisons test)

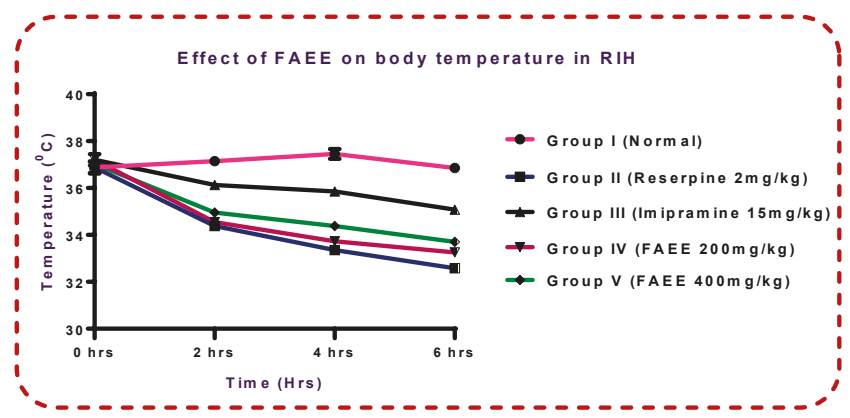

Graph 4. Effect of FAEE on body temperature in RIH.

All values were expressed as Mean $\pm \operatorname{SEM}(n=6)$. The data was analysised by One way ANOVA using Dunnett's multiple comparison tests; Significance at $\mathrm{aP}<0.05$, $\mathrm{bP}<0.01, \mathrm{cP}<0.001$ Non Significance (ns) at $\mathrm{P}>0.05$ Vs positive control.

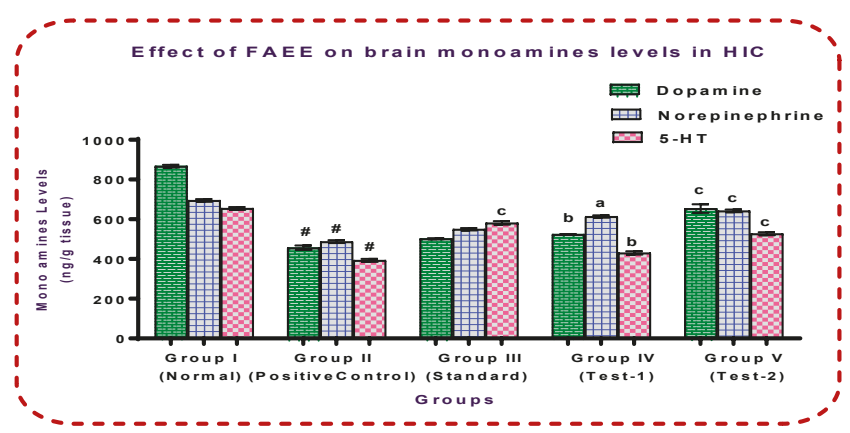

Graph 6. Effect of FAEE on brain monoamines levels in HIC.

All values were expressed as Mean $\pm \operatorname{SEM}(n=6)$. The data was analysised by One way ANOVA using Dunnett's multiple comparison tests; Significance at aP $<0.05$, $b P<0.01, c P<0.001$ Non Significance (ns) at $P>0.05$ Vs positive control.

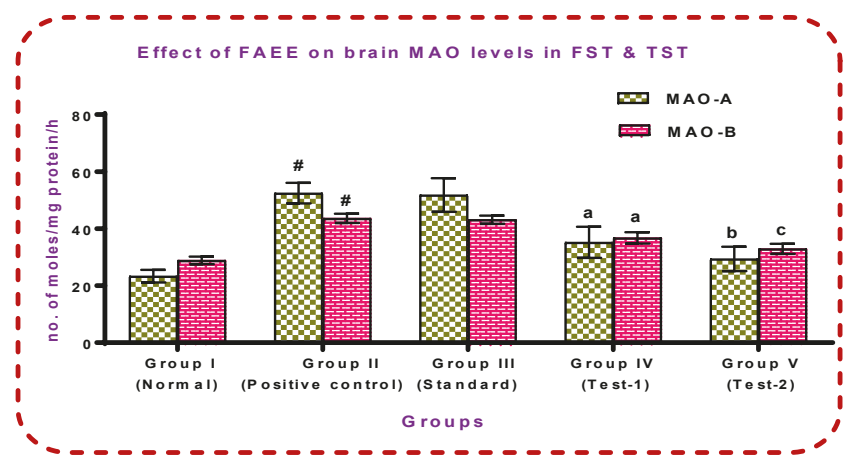

Graph 8. Effect of FAEE on brain MAO levels in FST and TST.

All values were expressed as Mean $\pm \operatorname{SEM}(n=6)$. The data was analysised by One way ANOVA using Dunnett's multiple comparison tests; Significance at $\mathrm{aP}<0.05$, $\mathrm{bP}<0.01, \mathrm{CP}<0.001$ Non Significance (ns) at $\mathrm{P}>0.05 \mathrm{Vs}$ positive control. 


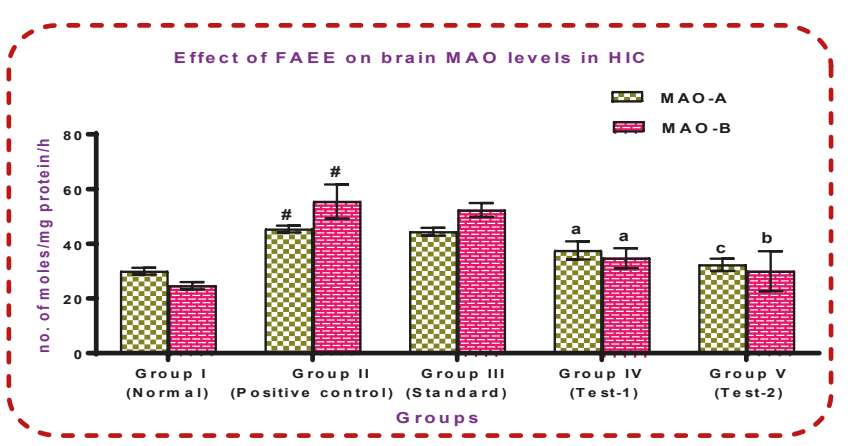

Graph 9. Effect of FAEE on brain MAO levels in HIC.

All values were expressed as Mean \pm SEM $(n=6)$. The data was analysised by One way ANOVA using Dunnett's multiple comparison tests; Significance at aP< 0.05 $\mathrm{bP}<0.01, \mathrm{cP}<0.001$ Non Significance (ns) at $\mathrm{P}>0.05$ Vs positive control.

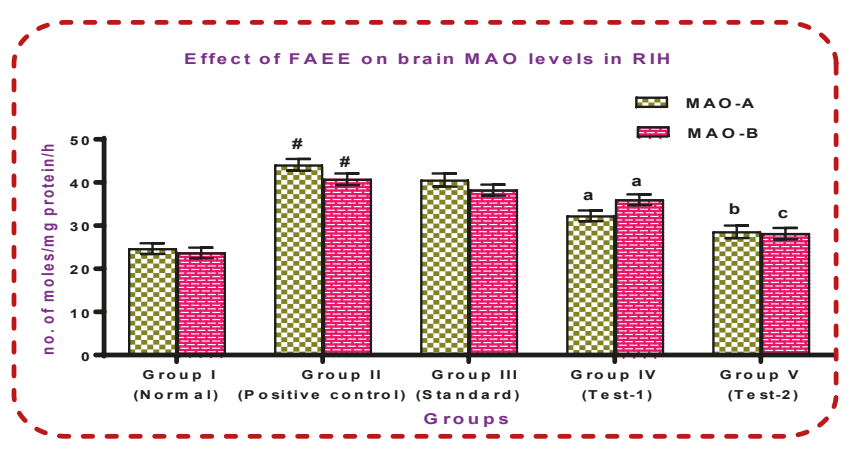

Graph 10. Effect of FAEE on brain MAO levels in RIH.

All values were expressed as Mean $\pm \operatorname{SEM}(n=6)$. The data was analysised by One way ANOVA using Dunnett's multiple comparison tests; Significance at aP $<0.05$, $b P<0.01, c P<0.001$ Non Significance (ns) at $P>0.05$ Vs positive control.

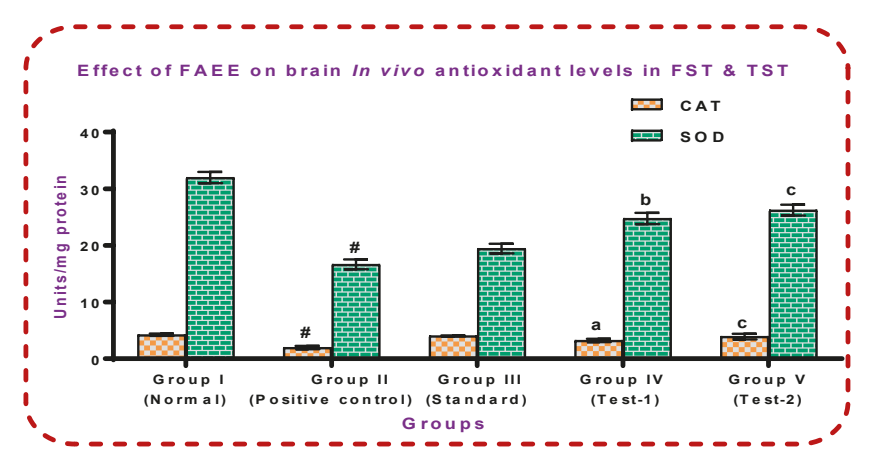

Graph 11. Effect of FAEE on brainin vivoantioxidant levels inFST and TST.

All values were expressed as Mean $\pm \operatorname{SEM}(n=6)$. The data was analysised by One way ANOVA using Dunnett's multiple comparison tests; Significance at $\mathrm{aP}<0.05$, $\mathrm{bP}<0.01, \mathrm{cP}<0.001$ Non Significance (ns) at $\mathrm{P}>0.05$ Vs positive control.

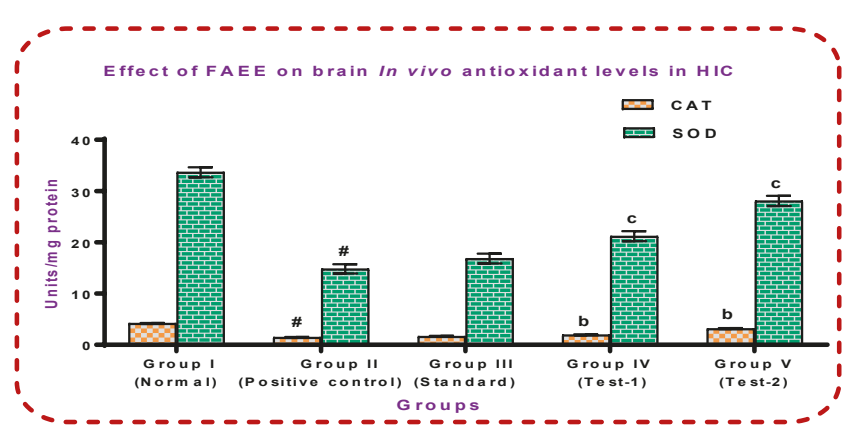

Graph 12. Effect of FAEE on brain in vivo antioxidant levels in HIC.

All values were expressed as Mean $\pm \operatorname{SEM}(n=6)$. The data was analysised by One way ANOVA using Dunnett's multiple comparison tests; Significance at $\mathrm{aP}<0.05$, $\mathrm{bP}<0.01, \mathrm{CP}<0.001$ Non Significance (ns) at $\mathrm{P}>0.05$ Vs positive control.

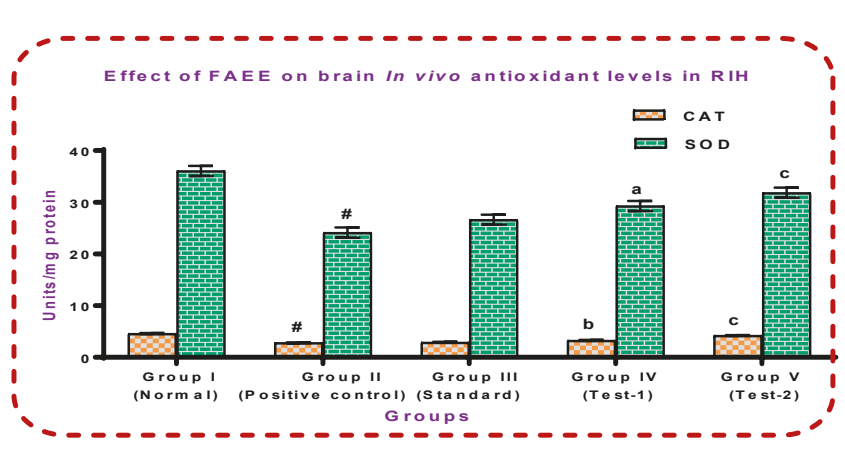

Graph 13. Effect of FAEE on brain in vivo antioxidant levels in RIH.

All values were expressed as Mean \pm SEM $(n=6)$. The data was analysised by One way ANOVA using Dunnett's multiple comparison tests; Significance at $\mathrm{aP}<0.05$, $b P<0.01, c P<0.001$ Non Significance (ns) at $P>0.05$ Vs positive control.
Table 1. Phytochemical constituents in FAEE

\begin{tabular}{clc}
\hline S.NO & Phytochemical constituents & Inference \\
\hline 1. & Carbohydrates & + \\
\hline 2. & Proteins \& amino acids & + \\
\hline 3. & Polysterols & - \\
\hline 4. & Anthraquinones & - \\
\hline 5. & Saponins & + \\
\hline 6. & Alkaloids & + \\
\hline 7. & Phenols & + \\
\hline 8. & Flavonoids & + \\
\hline 9. & Terpenoids & + \\
\hline 10. & Tannins & + \\
\hline
\end{tabular}

Present (+); Absent (-)

Table 2. Acute toxicity studies of FAEE

\begin{tabular}{lcccccc}
\hline Groups & $\begin{array}{c}\text { Dose/kg b.w } \\
\text { p.o }\end{array}$ & $\begin{array}{c}\text { Number } \\
\text { of animals }\end{array}$ & $\begin{array}{c}\text { Signs of } \\
\text { toxicity }\end{array}$ & $\begin{array}{c}\text { Onset of } \\
\text { toxicity }\end{array}$ & $\begin{array}{c}\text { Mortality } \\
\text { rate }\end{array}$ & $\begin{array}{c}\text { Duration } \\
\text { of study }\end{array}$ \\
\hline Group I & $5 \mathrm{mg} / \mathrm{kg}$ & 3 & $\begin{array}{c}\text { No signs } \\
\text { of toxicity }\end{array}$ & Nil & Nil & 14 days \\
\hline Group II & $50 \mathrm{mg} / \mathrm{kg}$ & 3 & $\begin{array}{c}\text { No signs } \\
\text { of toxicity }\end{array}$ & Nil & Nil & 14 days \\
\hline Group III & $300 \mathrm{mg} / \mathrm{kg}$ & 3 & $\begin{array}{l}\text { No signs } \\
\text { of toxicity }\end{array}$ & Nil & Nil & 14 days \\
\hline Group IV & $2000 \mathrm{mg} / \mathrm{kg}$ & 3 & $\begin{array}{l}\text { No signs } \\
\text { of toxicity }\end{array}$ & Nil & Nil & 14 days \\
\hline
\end{tabular}


Table 3. Effect of FAEE on duration of immobility in FST

\begin{tabular}{llccc}
\hline Groups & $\begin{array}{l}\text { Dose } \\
\text { (per kg b.w) }\end{array}$ & \multicolumn{2}{c}{ FST Immobility Time (Sec) } \\
\cline { 2 - 5 } & DAY 1 & DAY 7 & DAY 14 \\
\hline $\begin{array}{l}\text { Group I } \\
\text { (Normal) }\end{array}$ & Normal Saline 5ml & $11.4 \pm 3.835$ & $14 \pm 3.414$ & $16.8 \pm 2.393$ \\
\hline $\begin{array}{l}\text { Group II } \\
\text { (Positive } \\
\text { control) }\end{array}$ & Normal Saline 5ml & $185 \pm 5.414$ & $193.2 \pm 3.463$ & $200.6 \pm 6.749$ \\
\hline $\begin{array}{l}\text { Group III } \\
\text { (Standard) }\end{array}$ & Imipramine 15mg & $67 \pm 10.317^{\mathrm{c}}$ & $78.2 \pm 25.432^{\mathrm{c}}$ & $91.6 \pm 10.516^{\mathrm{c}}$ \\
\hline $\begin{array}{l}\text { Group IV } \\
\text { (Test-1) }\end{array}$ & FAEE 200mg & $121.6 \pm 10.03^{\mathrm{a}}$ & $134.4 \pm 29.85^{\mathrm{a}}$ & $147.6 \pm 15.15^{\mathrm{b}}$ \\
\hline $\begin{array}{l}\text { Group V } \\
\text { (Test-2) }\end{array}$ & FAEE 400mg & $106.2 \pm 10.87^{\mathrm{b}}$ & $113.5 \pm 15.30^{\mathrm{b}}$ & $138.2 \pm 12.58^{\mathrm{b}}$
\end{tabular}

(Test-2)

All values were expressed as mean \pm SEM, $(n=6)$. (One way ANOVA followed by Dunnett's multiple comparisons test)

Table 4. Effect of FAEE on duration of immobility in TST

\begin{tabular}{llccc}
\hline Groups & \multirow{2}{*}{$\begin{array}{l}\text { Dose } \\
\text { (per kg b.w) }\end{array}$} & \multicolumn{3}{c}{ TST Immobility Time (Sec) } \\
\cline { 2 - 5 } & DAY 1 & DAY 7 & DAY 14 \\
\hline $\begin{array}{l}\text { Group I } \\
\text { (Normal) }\end{array}$ & Normal Saline 5ml & $14.6 \pm 3.208$ & $17.6 \pm 1.927$ & $25.4 \pm 2.193$ \\
\hline $\begin{array}{l}\text { Group II } \\
\text { (Positive }\end{array}$ & Normal Saline 5ml & $166 \pm 5.098$ & $189.6 \pm 7.135$ & $198.6 \pm 5.542$ \\
control) & & & & \\
\hline $\begin{array}{l}\text { Group III } \\
\text { (Standard) }\end{array}$ & Imipramine15mg & $51 \pm 6.588^{c}$ & $55.4 \pm 3.694^{c}$ & $62 \pm 2.121^{c}$ \\
\hline $\begin{array}{l}\text { Group IV } \\
\text { (Test-1) }\end{array}$ & FAEE 200 mg & $133.8 \pm 10.03^{\mathrm{c}}$ & $157.6 \pm 9.78$ & $168.6 \pm 9.112^{\mathrm{b}}$ \\
\hline $\begin{array}{l}\text { Group V } \\
\text { (Test-2) }\end{array}$ & FAEE 400mg & $105.2 \pm 9.035^{c}$ & $120.6 \pm 4.631^{c}$ & $123.6 \pm 4.631^{c}$ \\
\hline
\end{tabular}

All values were expressed as mean \pm SEM, $(n=6)$. (One way ANOVA followed by Dunnett's multiple comparisons test)

Table 5. Effect of FAEE on duration of catalepsy in HIC

\begin{tabular}{llccc}
\hline Groups & $\begin{array}{l}\text { Dose } \\
\text { (per kg b.w) }\end{array}$ & \multicolumn{3}{c}{ Catalepsy Time (Sec) } \\
\cline { 2 - 5 } & & DAY 3 & DAY 5 & DAY 7 \\
\hline $\begin{array}{l}\text { Group I } \\
\text { (Normal) }\end{array}$ & Normal Saline5ml & $2.75 \pm 0.478$ & $3.25 \pm 0.25$ & $3.5 \pm 0.288$ \\
\hline $\begin{array}{l}\text { Group II } \\
\text { (positive } \\
\text { control) }\end{array}$ & Haloperidol 1mg & $235 \pm 9.595$ & $246 \pm 6.338$ & $258.8 \pm 4.398$ \\
\hline $\begin{array}{l}\text { Group III } \\
\text { (Standard) }\end{array}$ & Haloperidol 1mg & $104.8 \pm 2.315^{\mathrm{c}}$ & $107 \pm 9.670^{\mathrm{c}}$ & $111.5 \pm 13.41^{\mathrm{c}}$ \\
\hline $\begin{array}{l}\text { Group IV } \\
\text { (Test-1) }\end{array}$ & $\begin{array}{l}\text { Haloperidol 1mg } \\
\text { + FAEE 200mg }\end{array}$ & $185.3 \pm 15.91^{\mathrm{a}}$ & $189.3 \pm 19.98^{\mathrm{b}}$ & $209.3 \pm 19.56^{\mathrm{b}}$ \\
\hline $\begin{array}{l}\text { Group V } \\
\text { (Test-2) }\end{array}$ & $\begin{array}{l}\text { Haloperidol 1mg } \\
\text { + FAEE 40mg }\end{array}$ & $128.3 \pm 10.73^{\mathrm{c}}$ & $170.8 \pm 15.27^{\mathrm{b}}$ & $193.3 \pm 14.56^{\mathrm{b}}$ \\
\hline
\end{tabular}

All values were expressed as mean \pm SEM, $(n=6)$. (One way ANOVA followed by Dunnett's multiple comparisons test)
Table 6. Effect of FAEE on body temperature in RIH

\begin{tabular}{|c|c|c|c|c|c|}
\hline \multirow[t]{2}{*}{ GROUPS } & \multirow{2}{*}{$\begin{array}{l}\text { DOSE } \\
\text { (per kg b.w) }\end{array}$} & \multicolumn{4}{|c|}{ RECTAL TEMPERATURE $\left({ }^{\circ} \mathrm{C}\right)$} \\
\hline & & 0 HOURS & 2 HOURS & 4 HOURS & 6 HOURS \\
\hline $\begin{array}{l}\text { Group I } \\
\text { (Normal) }\end{array}$ & $\begin{array}{l}\text { Normal } \\
\text { saline5ml }\end{array}$ & $36.88 \pm 0.23$ & $37.45 \pm 0.170$ & $37.45 \pm 0.21$ & $36.85 \pm 0.15$ \\
\hline $\begin{array}{l}\text { Group II } \\
\text { (positive } \\
\text { control) }\end{array}$ & $\begin{array}{l}\text { Reserpine } \\
2 \mathrm{mg}\end{array}$ & $36.88 \pm 0.26$ & $34.38 \pm 0.16$ & $33.35 \pm 0.12$ & $32.58 \pm 0.15$ \\
\hline $\begin{array}{l}\text { Group III } \\
\text { (Standard) }\end{array}$ & $\begin{array}{l}\text { Reserpine } \\
2 \mathrm{mg}+ \\
\text { Imipramine } \\
15 \mathrm{mg}\end{array}$ & $37.20 \pm 0.25^{c}$ & $36.13 \pm 0.08^{c}$ & $35.85 \pm 0.02^{c}$ & $35.08 \pm 0.15^{c}$ \\
\hline $\begin{array}{l}\text { Group IV } \\
\text { (Test-1) }\end{array}$ & $\begin{array}{l}\text { Reserpine } \\
2 \mathrm{mg}+\text { FAEE } \\
200 \mathrm{mg}\end{array}$ & $37.20 \pm 0.14^{\mathrm{a}}$ & $34.55 \pm 0.15^{b}$ & $33.73 \pm 0.17^{a}$ & $33.25 \pm 0.15^{b}$ \\
\hline $\begin{array}{l}\text { Group V } \\
\text { (Test-2) }\end{array}$ & $\begin{array}{l}\text { Reserpine } \\
2 \mathrm{mg}+\mathrm{FAEE} \\
400 \mathrm{mg}\end{array}$ & $37.03 \pm 0.02^{c}$ & $34.95 \pm 0.08^{c}$ & $34.38 \pm 0.18^{b}$ & $33.70 \pm 6.56^{c}$ \\
\hline
\end{tabular}

All values were expressed as Mean $\pm \operatorname{SEM}(n=6)$. The data was analyzed by One way ANOVA using Dunnett's multiple comparison tests; Significance at ${ }^{\mathrm{a}} \mathrm{P}<0.05,{ }^{\mathrm{b}} \mathrm{P}<0.01,{ }^{\mathrm{C}} \mathrm{P}<0.001$ Non Significance (ns) at $P>0.05$ Vs positive control.

Table 7. Effect of FAEE in PNEIT

\begin{tabular}{lccc}
\hline GROUPS & $\begin{array}{c}\text { DOSE } \\
\text { (per kg b.w) }\end{array}$ & $\begin{array}{c}\text { DEATH } \\
\text { NUMBER OF } \\
\text { ANIMALS }\end{array}$ & $\begin{array}{c}\text { LETHALITY } \\
(\%)\end{array}$ \\
\hline $\begin{array}{l}\text { Group I } \\
\text { (Normal) }\end{array}$ & Normal Saline 5ml & 0 & 0 \\
\hline $\begin{array}{l}\text { Group II } \\
\text { (positive } \\
\text { control) }\end{array}$ & Norepinephrine 4mg & 0 & 0 \\
\hline $\begin{array}{l}\text { Group III } \\
\text { (Standard) }\end{array}$ & $\begin{array}{c}\text { Norepinephrine 4mg + } \\
\text { Imipramine 40mg }\end{array}$ & 6 & 100 \\
\hline $\begin{array}{l}\text { Group IV } \\
\text { (Test-1) }\end{array}$ & Norepinephrine 4mg + FAEE 200mg & 2 & 33.33 \\
\hline $\begin{array}{l}\text { Group V } \\
\text { (Test-2) }\end{array}$ & Norepinephrine 4mg + FAEE400mg & 5 & 83.33 \\
\hline
\end{tabular}

Table 8. Effect of FAEE on brain monoamines levels in FST and TST

\begin{tabular}{llccc}
\hline GROUPS & $\begin{array}{l}\text { DOSE } \\
\text { (per kg b.w) }\end{array}$ & \multicolumn{3}{c}{ MONOAMINES LEVELS (FST \& TST) } \\
\cline { 2 - 5 } & DP & NE & $5-H T$ \\
\hline $\begin{array}{l}\text { Group I } \\
\text { (Normal) }\end{array}$ & Normal Saline 5ml & $879.56 \pm 5.99$ & $696.53 \pm 6.79$ & $692.23 \pm 6.99$ \\
\hline $\begin{array}{l}\text { Group II } \\
\text { (positive } \\
\text { control) }\end{array}$ & Normal Saline 5ml & $386.60 \pm 8.92$ & $232.32 \pm 22.8$ & $466.36 \pm 8.96$ \\
\hline $\begin{array}{l}\text { Group III } \\
\text { (Standard) }\end{array}$ & Imipramine15mg & $642.65 \pm 10.7^{c}$ & $605.66 \pm 16.9^{c}$ & $658.66 \pm 6.69^{c}$ \\
\hline $\begin{array}{l}\text { Group IV } \\
\text { (Test-1) }\end{array}$ & FAEE 200 mg & $434.62 \pm 18.8^{\mathrm{a}}$ & $310.40 \pm 20.89^{\mathrm{a}}$ & $495.99 \pm 6.82^{\mathrm{a}}$ \\
\hline $\begin{array}{l}\text { Group V } \\
\text { (Test-2) }\end{array}$ & FAEE 400mg & $463.63 \pm 15.9^{\mathrm{b}}$ & $343.23 \pm 20.8^{\mathrm{c}}$ & $507.55 \pm 5.79^{\mathrm{b}}$ \\
\hline
\end{tabular}

$\overline{\text { All values weretexpressed as Mean } \pm S E M(n=6) . T h e ~ d a t a ~ w a s ~ a n a l y z e d ~ b y ~ O n e ~ w a y ~ A N O V A ~}$

All values were expressed as Mean $\pm \operatorname{SEM}(n=6)$. The data was analyzed by One way ANOVA using Dunnett's multiple comparison tests; Significance at ${ }^{a} P<0.05,{ }^{b} P<0.01,{ }^{C} P<0.001$ Non Significance (ns) at $P>0.05$ Vs positive control. 
Table 9. Effect of FAEE on brain monoamines levels in HIC

\begin{tabular}{llccc}
\hline GROUPS & \multirow{2}{*}{$\begin{array}{l}\text { DOSE } \\
\text { ( per kg b.w) }\end{array}$} & \multicolumn{3}{c}{ MONOAMINES LEVELS (HIC) } \\
\cline { 3 - 5 } & DP & NE & $5-H T$ \\
\hline $\begin{array}{l}\text { Group I } \\
\text { (Normal) }\end{array}$ & Normal Saline 5ml & $867.16 \pm 6.35$ & $694.64 \pm 6.86$ & $654.29 \pm 6.97$ \\
\hline $\begin{array}{l}\text { Group II } \\
\text { (positive } \\
\text { control) }\end{array}$ & Haloperidol 1mg & $456.61 \pm 6.89$ & $487.26 \pm 6.96$ & $392.76 \pm 7.68$ \\
\hline $\begin{array}{l}\text { Group III } \\
\text { (Standard) }\end{array}$ & Haloperidol 1mg & $502.24 \pm 5.36$ & $549.35 \pm 5.96$ & $581.64 \pm 8.69^{c}$ \\
\hline $\begin{array}{l}\text { Group IV } \\
\text { (Test-1) }\end{array}$ & $\begin{array}{l}\text { Haloperidol 1mg } \\
\text { + FAEE 200mg }\end{array}$ & $523.64 \pm 5.98^{\mathrm{b}}$ & $613.98 \pm 5.94^{\mathrm{a}}$ & $430.35 \pm 8.45^{\mathrm{b}}$ \\
\hline $\begin{array}{l}\text { Group V } \\
\text { (Test-2) }\end{array}$ & $\begin{array}{l}\text { Haloperidol 1mg } \\
\text { + FAEE 40mg }\end{array}$ & $653.67 \pm 6.93^{\mathrm{c}}$ & $642.34 \pm 6.78^{\mathrm{c}}$ & $527.16 \pm 7.65^{\mathrm{c}}$ \\
\hline
\end{tabular}

All values were expressed as Mean \pm SEM $(n=6)$. The data was analyzed by One way ANOVA using Dunnett's multiple comparison tests; Significance at ${ }^{\mathrm{a}} \mathrm{P}<0.05,{ }^{\mathrm{b}} \mathrm{P}<0.01,{ }^{\mathrm{C}} \mathrm{P}<0.001$ Non Significance (ns) at $P>0.05$ Vs positive control.

Table 11. Effect of FAEE on brain MAO levels in FST and TST

\begin{tabular}{llcc}
\hline GROUPS & $\begin{array}{l}\text { DOSE } \\
\text { (per kg b.w) }\end{array}$ & $\begin{array}{c}\text { MONOAMINE OXIDASE LEVELS } \\
\text { (FST \& TST) }\end{array}$ \\
\cline { 2 - 4 } & & \multicolumn{1}{c}{ MAO-A } & MAO-B \\
\hline $\begin{array}{l}\text { Group I } \\
\text { (Normal) }\end{array}$ & Normal Saline 5ml & $23.33 \pm 2.23$ & $28.86 \pm 1.35$ \\
\hline $\begin{array}{l}\text { Group II } \\
\text { (positive control) }\end{array}$ & Normal Saline 5ml & $52.45 \pm 3.62$ & $43.61 \pm 1.65$ \\
\hline $\begin{array}{l}\text { Group III } \\
\text { (Standard) }\end{array}$ & Imipramine15mg & $51.82 \pm 5.89$ & $43.14 \pm 1.46$ \\
\hline $\begin{array}{l}\text { Group IV } \\
\text { (Test-1) }\end{array}$ & FAEE 200 mg & $35.22 \pm 5.45^{\mathrm{a}}$ & $36.78 \pm 1.98^{\mathrm{a}}$ \\
\hline $\begin{array}{l}\text { Group V } \\
\text { (Test-2) }\end{array}$ & FAEE 400mg & $29.37 \pm 4.29^{\mathrm{b}}$ & $32.93 \pm 1.78^{\mathrm{c}}$ \\
\hline
\end{tabular}

All values were expressed as Mean \pm SEM $(n=6)$. The data was analyzed by One way ANOVA using Dunnett's multiple comparison tests; Significance at ${ }^{\mathrm{a}} \mathrm{P}<0.05,{ }^{\mathrm{b}} \mathrm{P}<0.01,{ }^{\mathrm{C} P}<0.001$ Non Significance (ns) at $P>0.05$ Vs positive control.

Table 13. Effect of FAEE on brain MAO levels in RIH

\begin{tabular}{|c|c|c|c|}
\hline \multirow[t]{2}{*}{ GROUPS } & \multirow{2}{*}{$\begin{array}{l}\text { DOSE } \\
\text { (per kg b.w) }\end{array}$} & \multicolumn{2}{|c|}{ MONOAMINE OXIDASE LEVELS (RIH) } \\
\hline & & MAO-A & MAO-B \\
\hline $\begin{array}{l}\text { Group I } \\
\text { (Normal) }\end{array}$ & Normal saline $5 \mathrm{ml}$ & $24.68 \pm 1.23$ & $23.69 \pm 1.23$ \\
\hline $\begin{array}{l}\text { Group II } \\
\text { (Positive control) }\end{array}$ & Reserpine $2 \mathrm{mg}$ & $44.09 \pm 2.36$ & $40.75 \pm 1.33$ \\
\hline $\begin{array}{l}\text { Group III } \\
\text { (Standard) }\end{array}$ & $\begin{array}{l}\text { Reserpine } 2 \mathrm{mg} \\
+ \text { Imipramine } 15 \mathrm{mg}\end{array}$ & $40.56 \pm 2.52$ & $38.25 \pm 1.26^{c}$ \\
\hline $\begin{array}{l}\text { Group IV } \\
\text { (Test-1) }\end{array}$ & $\begin{array}{l}\text { Reserpine } 2 \mathrm{mg}+ \\
\text { FAEE } 200 \mathrm{mg}\end{array}$ & $32.27 \pm 4.25^{\mathrm{a}}$ & $36.02 \pm 1.23^{a}$ \\
\hline $\begin{array}{l}\text { Group V } \\
\text { (Test-2) }\end{array}$ & $\begin{array}{l}\text { Reserpine } 2 \mathrm{mg}+ \\
\text { FAEE } 400 \mathrm{mg}\end{array}$ & $28.58 \pm 2.45^{b}$ & $28.16 \pm 1.32^{c}$ \\
\hline
\end{tabular}

All values were expressed as Mean \pm SEM $(n=6)$. The data was analyzed by One way ANOVA using Dunnett's multiple comparison tests; Significance at ${ }^{a} \mathrm{P}<0.05,{ }^{\mathrm{b} P}<0.01,{ }^{\mathrm{C} P}<0.001$ Non Significance (ns) at $P>0.05$ Vs positive control.
Table 10. Effect of FAEE on brain monoamines levels in RIH

\begin{tabular}{|c|c|c|c|c|}
\hline \multirow[t]{2}{*}{ GROUPS } & \multirow{2}{*}{$\begin{array}{l}\text { DOSE } \\
\text { (per kg b.w) }\end{array}$} & \multicolumn{3}{|c|}{ MONOAMINES LEVELS (RIH) } \\
\hline & & DP & NE & $5-\mathrm{HT}$ \\
\hline $\begin{array}{l}\text { Group I } \\
\text { (Normal) }\end{array}$ & Normal saline $5 \mathrm{ml}$ & $842.18 \pm 7.89$ & $645.73 \pm 9.86$ & $669.67 \pm 8.65$ \\
\hline $\begin{array}{l}\text { Group II } \\
\text { (positive } \\
\text { control) }\end{array}$ & Reserpine $2 \mathrm{mg}$ & $567.76 \pm 8.69$ & $454.82 \pm 9.86$ & $410.26 \pm 9.61$ \\
\hline $\begin{array}{l}\text { Group III } \\
\text { (Standard) }\end{array}$ & $\begin{array}{l}\text { Reserpine } 2 \mathrm{mg}+ \\
\text { Imipramine } 15 \mathrm{mg}\end{array}$ & $796.64 \pm 9.87^{c}$ & $582.19 \pm 8.96^{c}$ & $612.89 \pm 7.96^{c}$ \\
\hline $\begin{array}{l}\text { Group IV } \\
\text { (Test-1) }\end{array}$ & $\begin{array}{l}\text { Reserpine } 2 \mathrm{mg}+ \\
\text { FAEE } 200 \mathrm{mg}\end{array}$ & $605.46 \pm 9.75^{a}$ & $505.34 \pm 7.26^{b}$ & $450.34 \pm 8.29^{b}$ \\
\hline $\begin{array}{l}\text { Group V } \\
\text { (Test-2) }\end{array}$ & $\begin{array}{l}\text { Reserpine } 2 \mathrm{mg}+ \\
\text { FAEE } 400 \mathrm{mg}\end{array}$ & $621.37 \pm 8.65^{b}$ & $544.86 \pm 9.25^{c}$ & $599.98 \pm 7.96$ \\
\hline
\end{tabular}

All values were expressed as Mean \pm SEM $(n=6)$. The data was analyzed by One way ANOVA using Dunnett's multiple comparison tests; Significance at ${ }^{a} \mathrm{P}<0.05,{ }^{\mathrm{b}} \mathrm{P}<0.01,{ }^{\mathrm{C}} \mathrm{P}<0.001$ Non Significance (ns) at $P>0.05$ Vs positive control.

Table 12. Effect of FAEE on brain MAO levels in HIC

\begin{tabular}{|c|c|c|c|}
\hline \multirow[t]{2}{*}{ GROUPS } & \multirow[t]{2}{*}{$\begin{array}{l}\text { DOSE } \\
\text { (per kg b.w) }\end{array}$} & \multicolumn{2}{|c|}{$\begin{array}{c}\text { MONOAMINE OXIDASE LEVELS } \\
(\mathrm{HIC})\end{array}$} \\
\hline & & MAO-A & MAO-B \\
\hline $\begin{array}{l}\text { Group I } \\
\text { (Normal) }\end{array}$ & Normal Saline $5 \mathrm{ml}$ & $26.96 \pm 1.23$ & $24.68 \pm 1.32$ \\
\hline $\begin{array}{l}\text { Group II } \\
\text { (positive control) }\end{array}$ & Haloperidol $1 \mathrm{mg}$ & $45.37 \pm 1.25$ & $55.46 \pm 6.23$ \\
\hline $\begin{array}{l}\text { Group III } \\
\text { (Standard) }\end{array}$ & $\begin{array}{l}\text { Haloperidol } 1 \mathrm{mg} \\
+ \text { Fluoxetine } 5 \mathrm{mg}\end{array}$ & $44.39 \pm 1.45$ & $52.35 \pm 2.56$ \\
\hline $\begin{array}{l}\text { Group IV } \\
\text { (Test-1) }\end{array}$ & $\begin{array}{l}\text { Haloperidol } 1 \mathrm{mg} \\
+ \text { FAEE } 200 \mathrm{mg}\end{array}$ & $37.56 \pm 1.33^{a}$ & $36.73 \pm 3.62^{a}$ \\
\hline $\begin{array}{l}\text { Group V } \\
\text { (Test-2) }\end{array}$ & $\begin{array}{l}\text { Haloperidol 1mg } \\
+ \text { FAEE 400mg }\end{array}$ & $32.32 \pm 1.24^{c}$ & $29.98 \pm 7.3^{b}$ \\
\hline
\end{tabular}

All values were expressed as Mean \pm SEM $(n=6)$. The data was analyzed by One way ANOVA using Dunnett's multiple comparison tests; Significance at ${ }^{a} \mathrm{P}<0.05,{ }^{b} \mathrm{P}<0.01,{ }^{\mathrm{c} P}<0.001$ Non Significance (ns) at $P>0.05$ Vs positive control.

Table 14. Effect of FAEE on brain in vivo antioxidant levels in FST and TST

\begin{tabular}{llcc}
\hline GROUPS & $\begin{array}{l}\text { DOSE } \\
\text { (per kg b.w) }\end{array}$ & \multicolumn{2}{c}{ ANTIOXIDANT LEVELS(FST \& TST) } \\
\cline { 2 - 4 } & Normal Saline 5ml & $4.23 \pm 0.09$ & $31.98 \pm 0.98$ \\
\hline $\begin{array}{l}\text { Group I } \\
\text { (Normal) }\end{array}$ & Normal Saline 5ml & $1.98 \pm 0.09$ & $16.65 \pm 0.87$ \\
\hline $\begin{array}{l}\text { Group II } \\
\text { (Positive control) }\end{array}$ & & \\
\hline $\begin{array}{l}\text { Group III } \\
\text { (Standard) }\end{array}$ & Imipramine15mg & $4.02 \pm 0.10$ & $19.85 \pm 0.86$ \\
\hline $\begin{array}{l}\text { Group IV } \\
\text { (Test-1) }\end{array}$ & FAEE 200 mg & $3.23 \pm 0.09^{\mathrm{a}}$ & $24.76 \pm 0.99^{\mathrm{b}}$ \\
\hline $\begin{array}{l}\text { Group V } \\
\text { (Test-2) }\end{array}$ & FAEE 400mg & & \\
\hline
\end{tabular}

All values were expressed as Mean \pm SEM $(n=6)$. The data was analyzed by One way ANOVA using Dunnett's multiple comparison tests; Significance at ${ }^{\mathrm{a}} \mathrm{P}<0.05,{ }^{\mathrm{b}} \mathrm{P}<0.01,{ }^{\mathrm{C}} \mathrm{P}<0.001$ Non Significance (ns) at $P>0.05$ Vs positive control. 
Table 15. Effect of FAEE on brain in vivo antioxidant levels in HIC

\begin{tabular}{|c|c|c|c|}
\hline \multirow[t]{2}{*}{ GROUPS } & \multirow{2}{*}{$\begin{array}{l}\text { DOSE } \\
\text { (per kg b.w) }\end{array}$} & \multicolumn{2}{|c|}{ ANTIOXIDANT LEVELS (HIC) } \\
\hline & & CAT & SOD \\
\hline $\begin{array}{l}\text { Group I } \\
\text { (Normal) }\end{array}$ & Normal Saline 5ml & $4.17 \pm 0.0968$ & $33.67 \pm 0.97$ \\
\hline $\begin{array}{l}\text { Group II } \\
\text { (Positive control) }\end{array}$ & Haloperidol $1 \mathrm{mg}$ & $1.44 \pm 0.08$ & $14.82 \pm 0.90$ \\
\hline $\begin{array}{l}\text { Group III } \\
\text { (Standard) }\end{array}$ & $\begin{array}{l}\text { Haloperidol } 1 \mathrm{mg} \\
+ \text { Fluoxetine } 5 \mathrm{mg}\end{array}$ & $1.65 \pm 0.09$ & $16.83 \pm 0.97$ \\
\hline $\begin{array}{l}\text { Group IV } \\
\text { (Test-1) }\end{array}$ & $\begin{array}{l}\text { Haloperidol } 1 \mathrm{mg} \\
+ \text { FAEE } 200 \mathrm{mg}\end{array}$ & $1.95 \pm 0.08^{b}$ & $21.19 \pm 0.98^{b}$ \\
\hline $\begin{array}{l}\text { Group V } \\
\text { (Test-2) }\end{array}$ & $\begin{array}{l}\text { Haloperidol } 1 \mathrm{mg} \\
+ \text { FAEE 40mg }\end{array}$ & $3.16 \pm 0.09^{c}$ & $28.08 \pm 0.99^{c}$ \\
\hline
\end{tabular}

All values were expressed as Mean \pm SEM $(n=6)$. The data was analyzed by One way ANOVA using Dunnett's multiple comparison tests; Significance at ${ }^{a} \mathrm{P}<0.05,{ }^{\mathrm{b}} \mathrm{P}<0.01,{ }^{\mathrm{C}} \mathrm{P}<0.001$ Non Significance (ns) at $P>0.05$ Vs positive control.

\section{DISCUSSION}

The presented work evaluated the antidepressant activity of ethanolic extract of Ferula asafoetida (FA) oleo gum resins in both mice and rats. The oleo gum resins of FA were extracted from the resinous ducts of roots and rhizome, which have been used in folk medicine for the management of the following ailments - as an antihyperglycaemic, anticholesterolemic, antifungal, antihepatotoxic, antiinflammatory, antioxidant,smooth muscle relaxant, as an anti-diabetic, antihelmintic, antiparasitic, anticoagulant, and anticarcinogenic activity, which has been proved. It is also used in the management of depression, but its neuropharmacological mechanism on antidepressant-like action is unknown.

FA was found to have potent antioxidant activity [23] and neuroprotective activity [24] which has been proved. Based on this evidence, i.e., effectiveness against the ROS which is one of the major causes of depression, as well as neuroprotective activity, initiated the development of an alternative medicine from a herbal source. The presented study was performed based on the knowledge of the authors of the current study, in order to evaluate the antidepressantlike activities by different methods, e.g., FST, TST, HIC, RIH and PNEIT, followed by estimation of brain bioamine levels, MAO levels and in vivo antioxidant parameters, and finally histopathology.

Preliminary phytochemical investigation of FAEE showed positive results for carbohydrates, amino acids, alkaloids, phenols, flavonoids, terpenoides and tannins. In acute toxicity studies, i.e., performed based on the OECD Guidelines 423 of the CPCSEA, revealed that this extract did not show any lethality $\left(\mathrm{LD}_{50}\right)$ in mice up to $2,000 \mathrm{mg} / \mathrm{kg}$, which was observed for $48 \mathrm{hrs}$ and prolonged for up to 14 days. Based on the obtained data from toxicity studies of FAEE, the safe dose range was considered as $200 \mathrm{mg} / \mathrm{kg}$ and $400 \mathrm{mg} / \mathrm{kg}$ b.w, which can be used for subsequent studies.

FST is the method in which animals were conditioned with stressful situations, i.e., by placing a mouse in a cylinder with enough water so that it cannot touch the bottom with its hind paws. Because of this, the animal feels stressed, and to escape from these stressful situations the animal performs swimming, climbing, jumping and rolling to avoid the situations. Due to the lack of escape, the animal finally
Table 16. Effect of FAEE on brain in vivo antioxidant levels in RIH

\begin{tabular}{|c|c|c|c|}
\hline \multirow[t]{2}{*}{ GROUPS } & \multirow{2}{*}{$\begin{array}{l}\text { DOSE } \\
\text { (per kg b.w) }\end{array}$} & \multicolumn{2}{|c|}{ ANTIOXIDANT LEVELS(RIH) } \\
\hline & & CAT & SOD \\
\hline $\begin{array}{l}\text { Group I } \\
\text { (Normal) }\end{array}$ & Normal saline $5 \mathrm{ml}$ & $4.6 \pm 0.09$ & $36.06 \pm 0.98$ \\
\hline $\begin{array}{l}\text { Group II } \\
\text { (Positive control) }\end{array}$ & Reserpine $2 \mathrm{mg}$ & $2.81 \pm 0.10$ & $24.14 \pm 0.99$ \\
\hline $\begin{array}{l}\text { Group III } \\
\text { (Standard) }\end{array}$ & $\begin{array}{l}\text { Reserpine } 2 \mathrm{mg} \\
+ \text { Imipramine } 15 \mathrm{mg}\end{array}$ & $2.91 \pm 0.10$ & $26.63 \pm 0.97^{c}$ \\
\hline $\begin{array}{l}\text { Group IV } \\
\text { (Test-1) }\end{array}$ & $\begin{array}{l}\text { Reserpine } 2 \mathrm{mg} \\
\text { + FAEE } 200 \mathrm{mg}\end{array}$ & $3.28 \pm 0.09^{b}$ & $29.28 \pm 0.97^{a}$ \\
\hline $\begin{array}{l}\text { Group V } \\
\text { (Test-2) }\end{array}$ & $\begin{array}{l}\text { Reserpine } 2 \mathrm{mg} \\
+ \text { FAEE } 400 \mathrm{mg}\end{array}$ & $4.21 \pm 0.08^{c}$ & $31.85 \pm 0.99^{c}$ \\
\hline
\end{tabular}

All values were expressed as Mean \pm SEM $(n=6)$. The data was analyzed by One way ANOVA using Dunnett's multiple comparison tests; Significance at ${ }^{\mathrm{a} P}<0.05,{ }^{\mathrm{b}} \mathrm{P}<0.01,{ }^{\mathrm{C}} \mathrm{P}<0.001$ Non Significance (ns) at $P>0.05$ Vs positive control.

feels helpless and remains immobile, indicating depression which also occurs similarly in human daily life $[25,26]$.

The TST method is conceptually similar to that of the FST, but in which the stressful situation is induced by suspending the animal from a string attached to a metal stand by using adhesive tape placed $1 \mathrm{~cm}$ from the tip of the tail. The string was $58 \mathrm{~cm}$ above the tabletop. The stressful condition to the animal induces it to escape from this situation by continuous mobility. Due to the lack of escape from this situation, animal finally feels helpeless and it remains immobile, indicating depression which similarly occurs in human daily life $[27,28]$.

In the presented study, FST and TST were tested against the normal saline ( $10 \mathrm{ml} / \mathrm{kg}$ b.w p.o), and standard antidepressant drug (Imipramine $15 \mathrm{mg} / \mathrm{kg}$ b.w p.o) and FAEE at doses of $200,400 \mathrm{mg} / \mathrm{kg}$ b.w p.o for 14 days in mice (20-25g b.w). A significant decrease was observed in the immobility time in standard and FAEE treated animals, compared with the positive control animals, which indicates antidepressantlike activity.

The HIC method is also one of the depression screening methods which is based on the mechanism of action. In this method, oxidative stress was induced by administration of haloperidol for 7 days, i.e., by blocking the D2 receptor of the brain it induces catalepsy, i.e., due to the turnover of dopamine levels in the brain by metabolic process by MAO, and also in certain conditions haloperidol directly causes necrosis in brain cells which is induced by oxidative stress. Catalepsy duration was tested against the unknown drug. If a decrease in catalepsy time was found, it may possess the antidepressant-like activity. HIC standard treated (fluoxitine $5 \mathrm{mg} / \mathrm{kg}$ ) and FAEE treated animals showed a significant decrease in the catalepsy time, compared with the animals treated only with haloperidol [29].

Reserpine-induced hypothermia is the one of the most common methods for the evaluation of a new drug for antidepressant activity. Reserpine acts directly on the brain and depletes all monoamines present in the brain, which leads to hypothermia, indicating the depression of CNS. Evaluation was by inserting a rectal thermometer into the rectum of rat to a depth of $2 \mathrm{~cm}$. Reading were noted every $2 \mathrm{hr}$ for up to $6 \mathrm{hrs}$, i.e., at $0,2,4$ and $6 \mathrm{~h}$. FAEE and the standard drug (imipramine) showed a significant increase in temperature, compared with the positive control animals, i.e., those treated only with reserpine [30]. 
The norepinephrine-induced toxicity test is based on the mechanism of action, as already discussed above, and depletion of the monoamines (nor epinephrine and serotonin) levels in the brain may lead to a depressed condition. In this study, one of the monoamine - norepinephrine - was pretreated and the animals were post-treated against the unknown drug [test drug or standard drug] if they showed norepinephrine increasing activity that led to the death of the animal, caused by norepinephrine toxicity. That lethality indicated antidepressant activity [31,32].

PNEIT standard (Imipramine $40 \mathrm{mg}$ ) treated animals showed $100 \%$ lethality, FAEE $200 \mathrm{mg} / \mathrm{kg}$ b.w showed $33.33 \%$ lethality, and FAEE $400 \mathrm{mg} / \mathrm{kg}$ b.w showed $67 \%$ lethality, compared with the positive control animals. This indicates that FAEE has potent antidepressant- like activity that potential depends on the concentration gradient [33].

The brain is the major organ which consumes almost 20\% of total basal oxygen consumed by the whole body, which indicates that the brain is highly prone to oxidative stress. As discussed above, oxidative stress is one of the major reason for depression and damages the communication system between the two neurons by ischemic conditions in brain tissues; due the damage caused, the neuron monoamines levels are also decreases [34].

In vivo antioxidant studies performed for both externally induced stress animals, i.e., FST and TST performed animals and oxidative stress induce animals, i.e., HIC. In both tests, FAEE showed a significant increase in SOD and CAT levels, compared with the depression-induced (positive control) animals [35]. This effect may be due the presence of phytochemical constituents like 'Ferulic acid and umbellifedrone', which has already been reported [36] It may also be due to the neuroprotective activity of other phytoconstituents, such as flavonoids, phenolic acids and polysulfide compounds [37].

In brain monoamines and MAO levels parameters, the estimation FAEE has shown a significant increase in monoamine (serotonine, noradrenaline and dopamine) levels, and a potent decrease in MAO (MAO-A and MAO-B) levels in TST, HIC and RIH performed animals. FAEE at $400 \mathrm{mg} / \mathrm{kg}$ b.w showed a greater effect when compared with $200 \mathrm{mg} / \mathrm{kg}$ b.w., indicating that FAEE has potent dose dependent antidepressant-like activity [38].

\section{CONCLUSION}

From the obtained results it can be concluded that FAEE has potent antidepressant-like activity. This effect is may be due to the anti-oxidant property of Ferulic acid and umbelliferone, or may be due to the neuroprotective activity of other major phytoconstituents, such as flavonoids, phenolic acids and polysulfide compounds. To identify the particular compound responsible for the antidepressant-like activity requires further molecular level studies.

\section{Acknowledgements}

The authors wish to thank the management of Krishna Teja Pharmacy College, Chadalawada nagar, Tirupati, Chittoor District, Andhra Pradesh, India, for providing the necessary facilities.

\section{REFERENCES}

1.Sudhakar Pemminati, Gopalakrishna H.N, Ashok K Shenoy, Sudhanshu Sekhar Sahu, Shishir Mishra, Vinayak Meti, Nair Vinod. Antidepressant activity of aqueous extract of fruits of Emblicaofficinalis in mice. International Journal of Applied Biology and Pharmaceutical Technology 2010; 1(2): 449-454.

2. Ravindra C. Sutar, Sanjay B. Kasture, V.K. Kalaichelvan. Evaluation of antidepressant activity of leaf extracts of Holoptelea integrifolia (roxb) plant in experimental animals. Int J Pharm Pharm Sci. 2014; 6(6): 250-253.

3. Ginpreet Kaur, Mihir Invally, Resham Sanzagiri, Harpal S. Buttar. Evaluation of the antidepressant activity of Moringa oleifera alone and in combination with Fluoxetine. Journal of Ayurveda \& Integrative Medicine 2015; 6(4): 273-279.

4. Saldanha D, Kaspate, B. Karmarkar, K. Shah, S. Narkhede. Evaluation of antidepressant activity of ethanolic extract momordica charantia unripen fruit. International Journal of Pharmaceutical Sciences and Drug Research 2015; 7(1): 68-71.

5. Arvind Agarwal, Deepika Bora, Chanderpriya Agarwal, Ratendra Kumar and Veermaram Choudhary. CNS stimulant and antidepressant activity of seeds of Abelmoschus esculentus in rats. Bull Pharm Res. 2015; 5(2): 1-4.

6. Santosh P, Venugopl R, Nilakash AS, Kunjbihari S, Dr. Mangala L. Antidepressant Activity of methanolic extract of Passiflora foetida leaves in mice. International Journal of Pharmacy and Pharmaceutical Sciences $2011 ; 3(1)$ : 112-115.

7. Mishra Swati, Jena Monalisa, Pal Abhisek. Evaluation of antidepressant activity of Eclipta alba using animal models. Asian J Pharm Clin Res. 2013; 6(3): 118-120.

8. Porsolt RD, Anton G, Blavet N, Jalfre M. Behavioral despair in rats: a new model sensitive to antidepressant treatments. Eur J Pharmacol. 1978; 47: 379-381.

9. Saldanha, D Kaspate, B Karmarkar, K Shah, S Narkhede. Evaluation of antidepressant activity of ethanolic extract momordica charantia unripen fruit. International Journal of Pharmaceutical Sciences and Drug Research 2015; 7(1): 68-71.

10. Steru L, Chermat R, Thierry B, Simon P. The tail suspension test: a new method for screening antidepressants in mice. Psychopharmacology (Berl). 1985; 85: 367-70.

11. Jamwal Neetu Singh, Kumar Sunil, Rana A.C. Antidepressant activity of methanolic extract of Foeniculum vulgare (Fennel) fruits in experimental animal models. Journal of Applied Pharmaceutical Science 2013; 3(9): 65-70.

12. Pamminati, V. Nair, P. Dorababu, H.N. Gopala Krishna, M.R.S.M. Pai. Effect of ethanolic leaf extract of Ocimum sanctum on haloperidol induced catalepsy in albino mice. Inidian jorunal of pharmacology 2013; 39(2): 87-89.

13. Parle Milind and Gurditta. Papita Fruit: A Delicious Remedy for Depression. IJRAP. 2011; 2 (4): 1358-1364.

14. H.Grehard Vogel. Drug discovery and evalution. second eition, Springer 2012.

15. Sigg FB. Pharmacological studies with Tofranil. Canadian Psychiat A J. 1959; 4: 575.

16. Habibur Rahman, M.C Eswaraiah, Simple spectroscopic Methods for estimating Brain Neurotransmitters, Antioxidant Enzymes of Laboratory animals like Mice: A review. Pharmatutor Art. 1244, 2008.

17. Ajit Kumar Thakur, Shyam Sunder Chatterjee and Vikas Kumar. Antidepressant-like activity of Andrographis paniculata in type-2 diabetic rats. Clinical Pharmacology and Biopharmaceutics 2014; S2: 2-9.

18. Shao-Xiao Yan, Jun-Lian Lang, Yan-Ying Song, Yu-Ze Wu, MengHan Lv, Xia Zhao, Yan-Hong Liu and Chun-Yan Xu. Studies on antidepressant activity of four flavonoids isolated from Apocynum venetum linn (apocynaceae) leaf in mice. Tropical Journal of Pharmaceutical Research 2015; 14(12): 2269-2277.

19. Dharani Prasad, V. Ravi chandiran, K. Pavan Kumar, S. Kavimani and V. Suba. Pharmacological Evidences for The Antiamnesic Effect of Dalbergia Latifoia Roxb in Mice. IJPCBS. 2013; 3(3): 955-961.

20. Claiborne JY, Malinowski DP, Fridovich I. "Purification and characterization of hydroperoxidase II of Escherichia coli B”. J Biol Chem. 1979; 254(22): 11664-11668.

21. Mishra HP, Fridovich I. "The role of superoxide anion in the autooxidation of epinephrine and a simple assay for superoxide dismutase". J BiolChem 1972; 247: 3170-3175. 
22. Bancroft JD, Gamble M. Theory and Practice of Histological Techniques. Churchill Livingstone, 5th Edition. Harcourt Publisher Limited, London 2002, 153

23. Farshad Homayouni Moghadam, Maryam Dehghan, Ehsan Zarepur, Reyhaneh Dehlavi, Fatemeh Ghaseminia, Shima Ehsani, Golnaz Mohammadzadeh, Kazem Barzega. Oleo gum resins of Ferula assafoetida L. ameliorates peripheral neuropathy in mice. Journal of ethnopharmacology 2014; 154(1): 183-189.

24. Arshiya Sultana, Asma K, Khaleequr Rahman and Shafeequr Rahman. Oleo-gum-resins of Ferula asafoetida: A traditional culinary spice with versatile pharmacological activities. Research Journal of Recent Sciences 2015; 4: 16-22.

25. Santosh P, Venugopl R, Nilakash A S, Kunjbihari S, Dr. Mangala L. Antidepressant Activity of methanolic extract of Passiflora foetida leaves in mice. International Journal of Pharmacy and Pharmaceutical Sciences 2011; 3(1): 112-115.

26. Mishra Swati, Jena Monalisa, Pal Abhisek. Evaluation of antidepressant activity of Eclipta alba using animal models. Asian J Pharm Clin Res. 2013; 6(3): 118-120.

27. Steru L, Chermat R, Thierry B, Simon P. The tail suspension test: a new method for screening antidepressants in mice. Psychopharmacology (Berl). 1985; 85: 367-70.

28. Saldanha, D. Kaspate, B. Karmarkar, K. Shah, S. Narkhede. Evaluation of antidepressant activity of ethanolic extract momordica charantia unripen fruit. International Journal of Pharmaceutical Sciences and Drug Research 2015; 7(1): 68-71.

29. Jamwal Neetu Singh, Kumar Sunil, Rana A.C. Antidepressant activity of methanolic extract of Foeniculum vulgare (Fennel) fruits in experimental animal models. Journal of Applied Pharmaceutical Science 2013; 3(9): 65-70.

30. Parle Milind and Gurditta. Papita Fruit: A Delicious Remedy for Depression. IJRAP. 2011; 2(4): 1358-1364.

31. Singh G. K. And Kumar V. Neuropharmacological screeningand lack of antidepressant activity of Standardized extract of Fumaria indica. Electronic Journal of Pharmacology and Therapy 2010; 3: 19-28.

32. H.Grehard Vogel. Drug discovery and evalution, second eition, Springer 2012.

33. Sigg FB. Pharmacological studies with Tofranil. Canadian Psychiat A J. 1959; 4: 575.

34. Warner DS, Sheng H, Batinić-Haberle I. Oxidants, antioxidants and the ischemic brain. J Exp Biol. 2004; 207: 3221-3231.

35. Farshad Homayouni Moghadam, Maryam Dehghan, Ehsan Zarepur, Reyhaneh Dehlavi, Fatemeh Ghaseminia, Shima Ehsani, Golnaz Mohammadzadeh, Kazem Barzega. Oleo gum resins of Ferula assafoetida L. ameliorates peripheral neuropathy in mice. Journal of ethnopharmacology 2014; 154(1): 183-189.

36. Kareparamban, PH Nikam, AP Jadhav, VJ Kadam. Ferula foetida "Hing": A Review. 2012 RJPBCS. 2012; 3(2): 775-786.

37. Farshad Homayouni Moghadam, Behzad Vakili Zarch, Mohammad Shafie. Double edged effect of gum-resins of ferula assa-foetida on lifespan of neurons. Iranian journal of basic medical sciences 2013; 16(4): 660

38. Ajit Kumar Thakur, Shyam Sunder Chatterjee and Vikas Kumar. Antidepressant-like activity of Andrographis paniculata in type-2 diabetic rats. Clinical Pharmacology and Biopharmaceutics 2014; S2: 2-9. 